\title{
Additions et corrections à l'inventaire des Chironomidés (Diptera) de France depuis 1990
}

\author{
H. Laville ${ }^{1}$ \\ B. Serra-Tosio 2
}

Mots clés.: Diptera, Chironomidae, faunistique, potamal, France.

Une liste de «46» espèces de Chironomidés récoltées pour la première fois en France continentale est présentée. La moitié (22) proviennent des zones potamiques des fleuves Loire et Rhône. Des corrections et des compléments de répartition sont apportés au premier bilan faunistique (Serra-Tosio \& Laville 1991). Huit genres Hayesomyia, Dratnalia, Euryhapsis, Gillotia, Kloosia, Lipiniella, Robackia, Saetheria et un sous-genre Demicryptochironomus (Irmakia) sont signalés en France pour la première fois. Ces nouvelles récoltes (+ Parachironomus mauricii et Pseudomittia gracilis), portent à 646 les espèces de Chironomidés actuellement recensées sur le territoire français. Cela correspond a la moitié de la faune chironomidienne paléarctique connue.

Additions and corrections to the french chironomid (Diptera) inventory since 1990

Keywords : Diptera, Chironomidae, faunistic, potamal, France.

A list of «46» chironomid species recorded for the first time in continental France is provided. Half of them were found in the potamal zones of the rivers Loire and Rhone. Some corrections, recent synonyms and some distribution complements have been added to the previously published check-list (Serra-Tosio \& Laville 1991). Eight genera Hayesomyia, Dratnalia, Euryhapsis, Gillotia, Kloosia, Lipiniella, Robackia, Saetheria and one subgenus Demicryptochironomus (Irmakia) have been recorded for the first time in France. With these new records, (+ Parachironomus mauricii and Pseudomittia gracilis) 646 chironomid species are now recognised in France. This represents approximately half of the known palaearctic chironomid fauna.

\section{Introduction}

Un premier bilan des espèces de Chironomidae recensées en France depuis l'origine de leur étude avait permis de dénomber 598 espèces et 152 genres (SerraTosio \& Laville 1991) sur les quelque 1290 espèces répertoriées de la région paléarctique (Ashe \& Cranston 1990, Ashe 1992) réparties dans 187 genres (Ashe et al. 1987).

Depuis 1990 les communautés de Chironomidae des trois grands systèmes fluviaux Garonne, Loire et Rhône sont étudiées et notamment leurs zones potamiques, jusqu'ici peu ou pas prospectées.

1. Centre d'Ecologie des Systemes Aquatiques Continentaux, UMR C 5576 CNRS/UPS, Université Paul Sabatier, 118, route de Narbonne, F - 31062 Toulouse Cedex.

2. Universite Joseph Fourier, Centre de Biologie Alpine, Laboratoire Hydrosystèmes Alpins, BP 53, F - 38041 Gŕenoble Cedex 9.

Article available at http://www.limnology-journal.org or http://dx.doi.org/10.1051/limn/1996008
L'étude systématique de ce matériel par le premier auteur a révélé la présence de nouvelles espèces sur le territoire français. Elles font l'objet de cette liste complémentaire d'espèces qui récapitule également les nouvelles citations de la littérature parues après 1990 ou antérieurement omises et qui met à jour la taxonomie de quelques espèces du premier bilan faunistique.

\section{Principaux cours d'eau ou sites prospec- tés (Fig. 1)}

La distribution des communautés de Chironomidés est actuellement étudiée dans plusieurs grands réseaux hydrographiques français, dans le cadre de Diplôme d'Études Approfondies ou de Thèses d'Université : 1) à Lyon, dans le Rhône par Franquet (1996); 2) à Toulouse, dans la Garonne et ses affluents rive droite - Lez - Salat - (Gruarin 1993) et rive gauche - Save - par Céline Gruarin, dans la rivière Aude (Gendron 1996), dans la Loire et le Cher aux environs de Tours (Garcia 1993); 3) à Rennes, dans des cours d'eau d'un marais de Charente-Maritime, au sud de La Rochelle, face à l'île d'Oléron (Baudon 1995). 


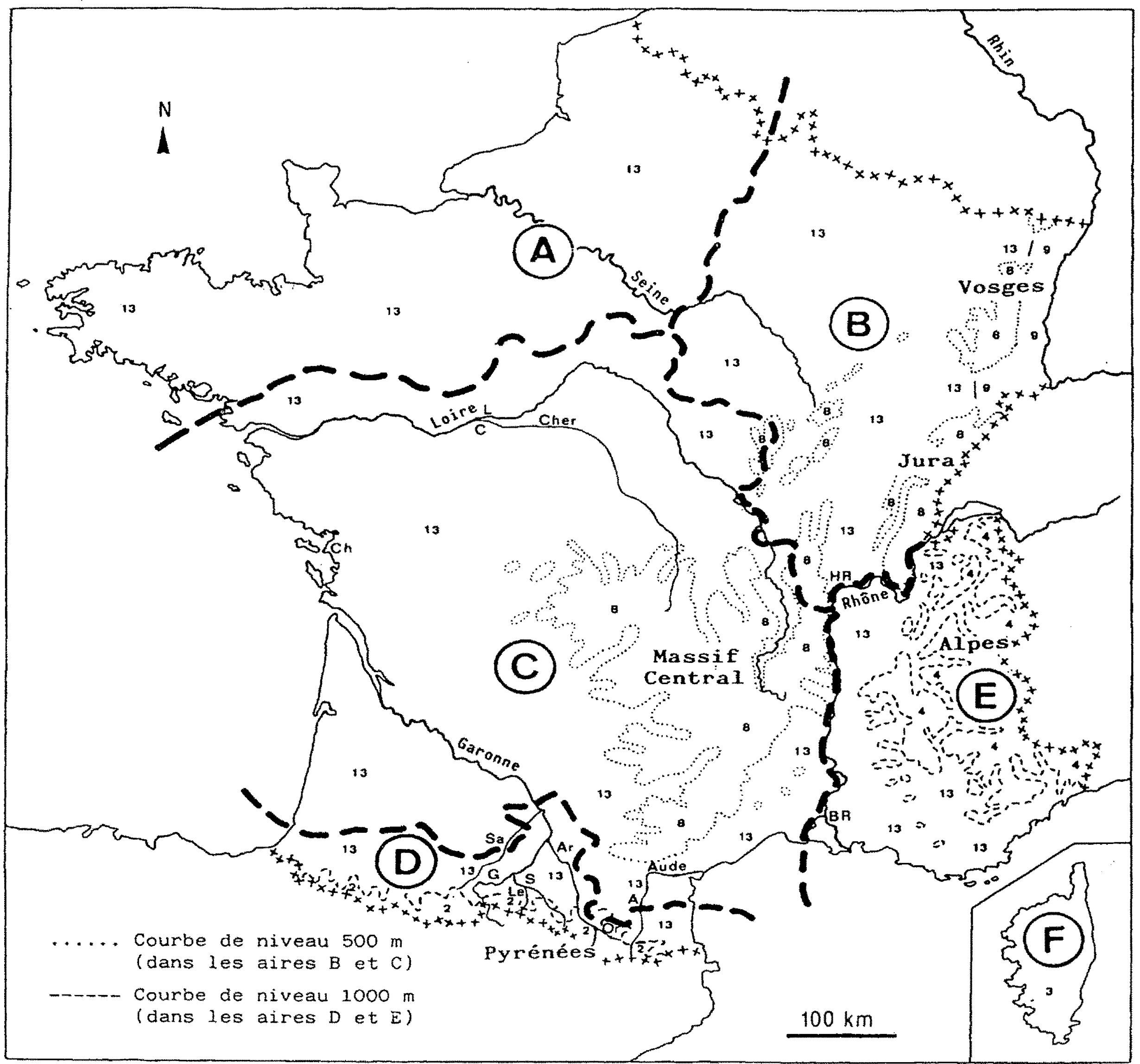

Fig 1. Découpage de la France : aire A (Bretagne, Normandie, nord de la France); aire B (est de la France, Lorraine, Alsace, Jura); aire C (Massif central et basses régions avoisinantes); aire D. (Pyrénées et basses régions avoisinantes); aire $\mathrm{E}$ (Alpes et basses régions avoisinantes); aire F (Corse). Les chiffres se rapportent aux régions de la Limnofauna Europaea (Illies 1978).

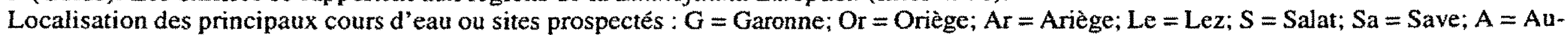
de; $\mathrm{BR}=$ bas Rhône; $\mathrm{HR}=$ haut Rhône; $\mathrm{C}=\mathrm{Cher} ; \mathrm{L}=\mathrm{Loire} ; \mathrm{Ch}=$ marais charentais.

Fig. 1. Geographical division of France : area A (Brittany, Normandy, North of France); area B (East of France, Lorraine, Alsace, Jura); area C (Central Massif and neighbouring lower regions); area D (Pyrenees and neighbouring lower regions); area $E$ (Alps and neighbouring lower regions); area F (Corsica). The numbers correspond to the regions of the Limnofauna Europaea (Illies 1978). Localization of the main sites and rivers studied. 
Les différents réseaux étudiés concernent la région 13 de la Limnofauna Europaea : ils se répartissent dans l'aire C pour la Loire, le Cher, le marais charentais, dans l'aire $\mathrm{D}$ pour la majeure partie du réseau de la Garonne et de l'Aude et dans l'aire E pour le Rhône (Fig. 1).

\section{Inventaire des nouvelles récoltes}

Les nouvelles espèces récoltées dans les différents milieux constituent la contribution essentielle de cette mise à jour faunistique des Chironomidés de France ; elles sont récapitulées dans le tableau 1.

Dans ce tableau sont également répertoriés les 10 espèces ou taxa nouveaux de la haute Ariège $(2100 \mathrm{~m}$ à $420 \mathrm{~m}$ ) (Griffon 1995) et de l'Oriège (900m; dét. J.M. Gendron), affluent et sous-affluent rive droite de la Garonne.

6 espèces également recensées dans des stations > $1000 \mathrm{~m}$ de l'Aude ou de l'Ariège s'inscrivent dans la région 2 de la Limnofauna et sont nouvelles pour les Pyrénées : elles sont marquées d'un astérisque dans la case correspondant à leur localisation dans le tableau 1 :

Eukiefferiella ancyla

Orthocladius ashei

Orthocladius cf. carlatus

Orthocladius vaillanti

Orthocladius ruffoi

(Aude : $1400 \mathrm{~m}$ )

(Aude : $1400 \mathrm{~m}$ )

(Aude 1700-1400m)

(Ariège : $1400 \mathrm{~m}$ )

(Aude : $1700-1400 \mathrm{~m}$ )

Micropsectra «pharetophora» (Aude : 1700-1400m)

Les noms d'espèces suivis d'un ${ }^{\circ}$ concernant des changements de nomenclature confirment une citation antérieure sans lieu de récolte précis (p. ex. Corynoneura gratias) ou une citation incertaine (p. ex. Rheotanytarsus ringei).

La plupart des études et des travaux recensés ici sont basés sur l'identification d'exuvies nymphales. Certains taxa suivis d'un signe + sont des types exuviaux définis par Langton (1991) mais encore sans dénomination linnéenne définie : ils ne sont pas décomptés comme nouveaux pour la France, du fait de leur position taxonomique encore hypothétique et de leur appartenance à des genres déjà signalés de France.

Par contre, trois types exuviaux encore indéfinis spécifiquement et appartenant aux genres Euryhapsis, Gillotia, ou au sous-genre Irmakia, signalés pour la première fois sur le territoire français, sont décomptés comme nouveaux taxa pour la France.

Les 46 espèces ou taxa nouveaux, dont une majorité d'Orthocladiinae (25) et de Chironomini (15), portent à 646 (Parachironomus mauricii Kruseman et Pseudosmitiia gracilis (G.) inclus) le total des espèces ou taxa actuellement recensés sur le territoire français, ce qui représente la moitié des espèces de Chironomidés connues de la région paléarctique.

Il faut noter cependant, que parmi les 598 espèces recensées dans le premier inventaire des Chironomidés de France (Serra-Tosio \& Laville 1991), 64 espèces, reprises de citations anciennes, sont considérées comme nomina dubia par Ashe \& Cranston (1990). Il est malheureusement probable qu'on ne connaîtra jamais le statut exact de ces espèces qui représentent tout de même $10 \%$ du recensement actuel.

Huit genres et un sous-genre sont nouveaux pour la faune de France :

1 Tanypodinae

2 Orthocladiinae

6 Chironomini
Hayesomyia Murray \& Fittkau 1985

Dratnalia Saether \& Halvorsen 1981, Euryhapsis Oliver 1981

Demicryptochironomus

(Irmakia Reiss 1988)

type 3 Reiss (dét. Langton)

Gillotia Kieffer 1921

Kloosia Kruseman 1933

Lipiniella Shilova 1961

Robackia Saether 1977

Saetheria Jackson 1977

Le Tanypodinae Hayesomyia et les Chironomini Kloosia, Lipiniella, Robackia, Saetheria sont des formes issues essentiellement des zones potamiques nouvellement prospectées.

Le genre Gillotia est connu des régions néarctique et afrotropicale et probablement néotropicale (Saether 1977, Pinder \& Reiss 1986). C'est sa première citation dans la région paléarctique : Ariège, Sinsat $(515 \mathrm{~m}): 1$ exuvie.

Nanocladius distinctus (Mallock) récoltée dans le Cher, est une espèce néarctique (Saether 1977) citée pour la première fois de la région paléarctique.

Deux Chironomini, Chironomus bonus des marais charentais et Lipiniella arenicola du bas Rhône, seulement connus de l'ex-Union soviétique (Ashe \& Cranston 1990), sont cités pour la première fois d'Europe.

Orthocladius cf. carlatus Roback (dét. P.H. Langton) : espèce néarctique (Oliver \& Dillon 1990). Première citation paléarctique en haute Bavière dans la rivière Isar (Reiss \& Reiff 1995). Deuxième citation paléarctique dans l'Aude : très fréquente à Pomas (140m) (Gendron 1996).

Paratanytarsus grimmii (Schneider) : première citation en France dans la rivière Cher de cette espèce parthénogénétique réputée pour être un véritable fléau. Vaste distribution : régions australopacifique, néotropicale, paléarctique. 
Tableau 1. Liste des nouvelles récoltes d'espèces ou taxa de Chironomidés avec leurs sites de captures :

G : Garonne, Or : Oriège ; Ar : Ariège; Le : Lez; $\mathbf{S}$ : Salat; Sa : Save

$\mathrm{BR}$ : bas Rhône; HR : haut Rhône

$C$ : Cher ; $\mathrm{L}$ : Loire ; $\mathrm{Ch}$ : marais charentais

Table 1. List of new records of chironomid species or taxa with their sampling sites.

\begin{tabular}{|c|c|c|c|c|c|c|c|c|c|c|c|c|}
\hline Espèces & G; & Or & Ar & l.e & $s$ & sa & $\Delta$ & $\mathbf{B R}$ & IIR & C & L. & Ch \\
\hline \multicolumn{13}{|l|}{ Tanypodinae (1) } \\
\hline Conchapelopia Pel (Langton 91) + & & & & & & & & & $\mathrm{x}$ & & & \\
\hline Ilayesomyia tripunctata (G.) & & & & & & & & $\mathrm{x}$ & $\mathrm{x}$ & $\mathrm{x}$ & $x$ & \\
\hline Paramerina Pel cf. cingulata (Langton 91$)^{\circ}$ & & $\mathrm{x}$ & & & & & & & $\mathrm{x}$ & & $\cdot$ & \\
\hline \multicolumn{13}{|l|}{ Diamesinae } \\
\hline Potthastia Pe 1 (Langton 91) + & $\mathrm{x}$ & & & & & $x$ & & & & & & \\
\hline \multicolumn{13}{|l|}{ Orthocladiinae (25) } \\
\hline Corynoneura gratias Schlee ${ }^{\circ}$ & & & & & & $\mathrm{x}$ & & & & & & \\
\hline Corynoneura minuscula $\mathrm{Br}$. & & & & & & & & & & & & $\mathrm{x}$ \\
\hline Cricotopus (Cricot.) caducus Hirv. & & & & & & & & & & & & $\mathrm{x}$ \\
\hline Cricotopus (lsocl.) brevipalpis K. & & $\mathrm{x}$ & & & & & & & & & & \\
\hline Cricotopus (Isocl.) intersectus (Staeg.) & & & & & & & & $\mathrm{x}$ & & $\mathrm{x}$ & & $\mathrm{x}$ \\
\hline Cricotopus (Isocl.) Pe 2 (Langton 91)+ & $\mathrm{x}$ & & & & & & & & & & & \\
\hline Dratnalia potamophylaxi (Fitt. \& Lell.) & & $\mathrm{x}$ & & & & & & & & & & \\
\hline Eukiefferiella ancyla Svensson & $\underline{x}$ & & $\mathrm{x}$ & $\mathrm{x}$ & $\mathrm{x}$ & & $\mathrm{x}^{*}$ & & & & & \\
\hline Euryhapsis Pe 3 (Langton 91) & & & & & & & & & $\mathrm{x}$ & & & \\
\hline Limnophyes edwardsi Saether & & & & & & $\mathrm{x}$ & & & & & & \\
\hline Limnophyes punctipennis (G.) & & & & & & $x$ & & & & & $\mathrm{x}$ & \\
\hline Nanocladius distinctus (Mall.) & & & & & & & & & & $\mathrm{x}$ & & \\
\hline Orthocladius (Euortho.) ashei Soponis & & & $\mathrm{x}^{*}$ & & & & $\mathrm{x}^{*}$ & & & & & \\
\hline Orthocludius (Euortho.) Lutcipes G. & & & & $\mathrm{x}$ & $\mathbf{x}$ & & & & & & & \\
\hline Orthocladius (Ortho.) cf. carlatus Roback & & & & & & & $\mathrm{X}^{*}$ & & & & & \\
\hline Orthocladius (Ortho.) pedestris K. & $\underline{x}$ & & $\underline{x}$ & $x$ & $x$ & & $\mathbf{x}$ & $\mathrm{x}$ & $\mathbf{x}$ & $\mathbf{x}$ & $\mathrm{x}$ & \\
\hline Orthocladius (Ortho.) rivinus $\mathrm{K}$. & & & & & & & & & & $\mathrm{x}$ & $\mathrm{x}$ & \\
\hline Orthocladius (Ortho.) vaillanti Lang. \& Crans. & $\mathrm{x}$ & & $\mathbf{x}^{*}$ & $\mathrm{x}$ & $\mathrm{x}$ & & & & & & & \\
\hline Orthocladius ruffoi Rossaro 1991 & $\mathrm{x}$ & $\mathbf{x}$ & $\mathrm{x}$ & & & & $x^{*}$ & $\underline{x}$ & & & & \\
\hline Parachaetocladius abnobeus (Wülk.) & & & & & & & $\mathrm{x}$ & & & & & \\
\hline Parakiefferiella pyrenaica Moubayed 1991 & & $\underline{x}$ & & & & & & & & & & \\
\hline Parakiefferiella smolandica (Br.) & & & & & & $\mathrm{x}$ & & $\mathbf{x}$ & & & & \\
\hline Parakiefferiella wuelkeri Moubayed 1994 & & & & & & & & $\underline{x}$ & & & & \\
\hline Psectrockadius (s. str.) brelmi $\mathrm{K}$. & & & & & & & & $\mathbf{x}$ & & $\mathbf{x}$ & & \\
\hline Psectrocladius (s. str.) fennicus Stora & & & & & & & & & & & & $\mathrm{x}$ \\
\hline
\end{tabular}


Tableau 1 Suite

Table 1. Continued.

\begin{tabular}{|c|c|c|c|c|c|c|c|c|c|c|c|c|}
\hline Stations & G & Or & $\Delta r$ & Le & $\underline{s}$ & $\underline{S \mathbf{a}}$ & $A$ & BR & IIR & $\mathrm{C}$ & $\mathrm{L}$ & Ch \\
\hline Psectrocladius (s. str.) oxyura Langton & & & & & & & & & & & $\mathrm{x}$ & \\
\hline Pseudosmittia oxoniana $\left(\right.$ Edw.) ${ }^{\circ}$ & & & & & & & $\mathrm{x}$ & & & & & \\
\hline Pseudosmittia Pe 2 (Langton 91) + & & & & & & & & & & $\mathrm{x}$ & & \\
\hline Thienemannia fulvofasciatus $(\mathrm{K})$. & & & & & & $\mathrm{x}$ & & & & & & \\
\hline Thienemannia $\mathrm{Pe} 2$ a (Langton 91) ${ }^{\circ}$ & $\mathrm{x}$ & & & & & $\mathrm{x}$ & & & & $\mathrm{x}$ & $\mathrm{x}$ & \\
\hline \multicolumn{13}{|l|}{ Chironominae Chironomini (15) } \\
\hline Chironomus bonus Shil. \& Dzhva. & & & & & & & & & & & & $\mathrm{x}$ \\
\hline Chironomus nuditarsis Keyl & & & & & & & $\mathrm{x}$ & $\mathrm{x}$ & & $\mathrm{x}$ & & $\mathrm{x}$ \\
\hline Chironomus prasinus $\mathrm{Mg}$. & & & & & & & & & & & & $\mathrm{x}$ \\
\hline Cryptotendipes usmaensis (Pag.) & & & & & & & & $\underline{x}$ & & $\mathrm{x}$ & $\underline{x}$ & \\
\hline Cryptotendipes Pe $1 \mathrm{c}$ (Langton 84$)+$ & & & & & & $x$ & & & & & & \\
\hline Demicryptochironomus (Irmakia) sp. 3 Reiss & $\mathrm{X}$ & & $\mathrm{x}$ & & & & & $\mathrm{x}$ & $\mathrm{x}$ & & & \\
\hline Dicrotendipes fusconotatus (K.) & & & & & & & & & & & & $\mathrm{x}$ \\
\hline Gillotia K.sp. & & & $\mathrm{x}$ & & & & & & & & & \\
\hline Glyptotendipes (s. str.) imbecillis (Walk.) & & & & & & & & & & & & $\mathrm{x}$ \\
\hline Glyptotendipes (s. str.) signatus: K. & & & & & & & . & $\underline{x}$ & & & & \\
\hline Kloosia pusilla (L.) & & & & & & & & $\mathrm{x}$ & & & & \\
\hline Lipiniella araenicola Shil. & & & & & & & & $\mathrm{x}$ & & & & \\
\hline Phaenopsectra "Pe f. Bala" (Langton 91) +. & & & & & & $x$ & & & & & & \\
\hline Polypedilum (Tripodura) acifer Townes & & & & & & & & & & $\mathrm{x}$ & $\mathrm{x}$ & \\
\hline Polypedilum (Tripodura) bicrenatum $\mathrm{K}$. & $\mathrm{x}$ & & & & & & $\underline{x}$ & & & & & \\
\hline Robackia demeijerei (Krus.) & & & & & & & $x$ & & $\mathbf{x}$ & $\mathrm{x}$ & $x$ & \\
\hline Saetheria reissi Jackson & & & & & & & & & . & $\mathrm{x}$ & $\mathrm{x}$ & \\
\hline \multicolumn{13}{|l|}{ Chironominae Tanytarsini (5) } \\
\hline Cladotanytarsus pallidus $\mathrm{K}$. & & & & & & & $\mathrm{x}$ & & & & & \\
\hline Cladotanytarsus Pe 6 (Langton 91) + & & & & & & & & & & $\mathrm{x}$ & & \\
\hline Krenopsectra fallax Reiss & & $\mathbf{x}$ & & & & & & & & & & \\
\hline Micropsectra lacustris Säw. & & & & & & & & & & & $x$ & \\
\hline Micropsectra "pharetophora" (Langton 91) + & & & $\mathbf{X}^{*}$ & & & & & & & & & \\
\hline Paratanytarsus grimmii (Schneider) & & & & & & & & & & $\mathrm{x}$ & & \\
\hline Paratanytarsus dissimilis Johannsen ${ }^{\circ}$ & & & & & & & & & & $\underline{x}$ & $\mathrm{X}$ & \\
\hline Rheotanytarsus rhenanus Lehm. ${ }^{\circ}$ & $\mathrm{x}$ & $\underline{x}$ & & & $\underline{x}$ & $x$ & & & $x$ & $\mathrm{x}$ & & \\
\hline Stempellina almi $\mathrm{Br}$. & & & & & & $\mathrm{x}$ & & $\underline{x}$ & & $\mathrm{x}$ & & \\
\hline Tanytarsus Pe 5a (Langton 91) + & $\mathrm{x}$ & & & & & & & & & & & \\
\hline
\end{tabular}


Plusieurs exuvies ou nymphes n'ont pu être identifiées spécifiquement ni assimilées à des formes définies par Langton (1991) : elles nécessitent la recherche de nymphes matures pour préciser leur taxonomie.

Elles appartiennent aux genres :

Monodiamesa sp. (Garonne)

Syndiamesa sp. (Ariège)

Parametriocnemus sp. (Ariège)

Polypedilum nov. pe. (Save : Montmaurin 1E : 26.08.90; Aude : Carcassonne $13 \mathrm{E}:$ 17.05.89).

Autres localisations d'espèces du tableau 1

Psectrocladius (Ps.) oxyura

Pyrénées orientales : Lac Vinça et rivière Têt, leg. $H$. Laville, dét. P.H. Langton.

\section{Chironomus nuditarsis}

Pyrénées orientales : Lac Vinça : leg. H. Laville, dét. P.H. Langton.

\section{Espèce nouvelle de la littérature}

Parachironomus mauricii (Kruseman 1933): syn. Chironomus varus limnaei Guibé 1942 (Reiss 1995). Signalée de la zone A13 : marais de Troarn (Calvados).

Parachironomus varus (G.) cité de France sans localisation précise par Lehmann (1970) et répertorié par Serra-Tosio \& Laville (1991) correspond (certainement) à ce même site du Calvados étudié par Guibé (1942 : sub Chironomus varus varus Goetghebuer).

Espèce oubliée dans Serra-Tosio \& Laville (1991) Pseudomittia gracilis (G.) E13 (7a)

\section{Mise à jour taxonomique}

Brillia flavifrons Johannsen (syn. longifurca K.) (Langton 1991).

Metriocnemus eurynotus (Holmgren) : (syn, obscuripes Holmgren) (Saether 1995).

Orthocladius ashei Soponis : les identifications de matériel nymphal sub $O$. rivicola $\mathrm{K}$. d'après Langton (1984) sont à revoir; elles peuvent modifier la répartition géographique de $O$. rivicola donnée par Serra-Tosio \& Laville (1991).

Orthocladius dentifer Brundin, sub $\mathrm{Pe} 4$ (Langton 1991) : les identifications de matériel nymphal sub $O$. oblidens, d'après Langton 1984, sont à revoir.

Orthocladius obumbratus Johannsen (syn. excavatus Brundin) (Langton \& Cranston 1991).

Orthocladius ruffoi Rossaro (= Rheorthocladius sp. A Thienemann 1944).

Pseudomittia oxoniana (Edw.) (syn. recta Edw.), (Langton, in litt).
Paratanytarsus dissimilis Johannsen (syn. confusus Palmen (Langton et al. 1990).

Rheotanytarsus rhenanus Klink : sub? ringei Lehman (Laville \& Serra-Tosio 1991) : Massif Central : C8, C13 : rivières Agoût, Aveyron, Lot, Tarn et probablement Pyrénées : ? D13 : Neste d'Aure inférieure et rio Llobregat (Espagne).

\section{Compléments de répartition}

Macropelopia notata : ajouter (17a) après A13

Procladius crassinervis : ajouter? A13(15) (17a)

Psectrotanypus varius : ajouter (17a) après A13

Prodiamesa olivacea : ajouter (7a) après E13.

Brillia modesta : ajouter (7a) après E13

"Chaetocladius» dentiforceps : ajouter (17a)

après $\mathrm{A} 13$

Chaetocladius mercieri : ajouter (57a) à A13

Clunio marinus : ajouter E13 (62b)

Eukiefferiella brevicalcar : ajouter (7a) après E13

Eukiefferiella claripennis : ajouter (7a) après E13

Halocladius variabilis : ajouter (57a) après ? E13

Limmophyes difficilis : ajouter E13 (7a)

Limmophyes minimus : ajouter (17a) après A13

Limmophyes pentaplastus : ajouter (7a) après E13

Metriocnemus obscuripes : ajouter (7a) après E13

Orthocladius fusiformis : ajouter (7a) après E13

Parakiefferiella gracillima : ajouter E13 (7a)

Paratrichocladius rufiventris : ajouter (7a)

après E13

Smittia rupicola : ajouter (57a)

Synorthocladius semivirens : ajouter (7a) après E13

Tvetenia calvescens : ajouter (7a) après E13

Chironomus aprilinus : ajouter (62a) après E13

Chironomus salinarius : ajouter (62a) après $\mathrm{C} 13$

Polypedilum nubeculosum : ajouter (17a) après A13

Micropsectra fusca : ajouter (17a) après A13

Tanytarsus pallidicornis : ajouter (17a) après A13

\section{Conclusion}

L'analyse de ces résultats faunistiques souligne l'intérêt de l'étude des zones potamiques les moins perturbées des grandes rivières de France - Rhône, Loire, Garonne - encore susceptibles d'abriter un peuplement spécifique de référence comparativement à celles d'autres grandes rivières ou fleuves européens - Danube, Rhin, Tamise - dont les peuplements chironomidiens sont certainement plus modifiés. 
Ces compléments à la première liste des Chironomidés de France montrent que des recherches faunistiques sur des groupes choisis peuvent, aujourd'hui encore, améliorer la connaissance de la biodiversité sur notre territoire et déboucher sur des résultats taxonomiques et biogéographiques précieux.

\section{Remerciements}

Les auteurs remercient les étudiants de DEA, Céline Gruarin, Frédéric Griffon (Toulouse) et les thésards Jean-Marie Gendron, Xavier-François Garcia (Toulouse), Claudine Baudon (Rennes), Evelyne Franquet (Arles) pour leur autorisation de rassembler les nouveautés faunistiques de leurs études. Nous remercions Bernard Cellot (Lyon) pour le legs du matériel du haut Rhône. Un grand merci à Peter Langton pour la vérification ou la détermination de plusieurs taxa lors de son séjour à Toulouse en août 1994 et à Declan Murray pour ses corrections du résumé anglais et pour ses conseils.

Travaux cités

Ashe P. 1992. - Corrections to the Chirmomidae part of the catalogue of palaearctic Diptera. Nether funds Journal of Aquatic Eco$\log y, 26(2-4): 215-221$.

Ashe P. \& Cranston P.S. 1990. - Family Chironominae. In Soos A. \& Papp L. : Catalogue of Palaearctic Diptera. Psychodidae - Chironomidae. Vol 2 : 113-355, Akademiai Kiado, Budapest.

Ashe P., Murray D.A. \& Reiss F. 1987. - The zoogeographical distribution of Chironomidae (Insecta : Diptera). Annls Limnol, 23 : 27-60.

Ashe P. \& O'Connor 1994. - Eukiefferiella ancyla and Paratanytarsus grimmii (Diptera : Chironomidae), new to Ireland, with a second record for Orthocladius (Symposiocladius) lignicola. Ir. Nat. J., $24: 364-366$.

Baudon C. 1995. - Ecologie des Chironomidae (Diptera, Nematocera) d'un marais charentais : structure spécifique, phénologie et densité des populations d'adultes. Thèse Université Rennes $1, \mathrm{n}^{\circ}$ $1113: 170 p+27$ annexes.

* (7a) Chouteau F. 1968. - Influence de la pollution industrielle et domestique sur les populations animales de la rivière Isère au cours de la traversée de la région grenobloise. Trav. Lab. Hydrobiol. Grenoble, 59-60:39-63.

* (17a) Ducrotoy J.P. 1976. - Stratégies démographiques des pop̄ulations de Chironomidés (Diptères) d'une mare semi-permanente du massif forestier de Paimpont (Ille-et-Vilaine). Thèse 3ème cycle, Université de Tours : $149 \mathrm{p}$.

Franquet E. 1996. - Occupation d'un espace fluvial par les Dipteres Chironomidés à l'aval du Rhône : répartition des espèces selon la nature du substrat et les conditions de débit. Thèse Université Lyon $1: 128 \mathrm{p}+$ annexes.

Garcia X.F. 1994. - Premier inventaire faunistique des Chironomidés (Diptera) du potamal de la Loire et du Cher (région de Tours). DEA Ecologie des Systèmes Aquatiques Continentaux, UPS Toulouse : 35 p. +3 annexes.

Gendron J.M. 1996. - Les Chironomidês (Diptera) de l'Aude, rivière méditerranéenne des Pyrénées orientales. Impact d'une crue catastrophique. Thèse Université, UPS Toulouse : $196 \mathrm{p}$.

Gruarin C. 1993. - Evaluation de l'impact des rejets des papeteries sur les peuplements de Chironomidés (Diptera) des rivières $\mathrm{Ga}$ ronne (Saint-Gaudens) et Salat (Saint-Girons). DEA Ecologie des Systèmes Aquatiques Continentaux, UPS Toulouse : 60 p. + annexes.

Griffon F. 1995. - Etude préliminaire des peuplements de Chironomidae (Diptera) comme bioindicateurs de la qualité des eaux de la haute Ariège. DESU, UPS Toulouse : $49 \mathrm{p}$ + annexes.
Guibé J. 1942. - Chironomes parasites de Mollusques Gastropodes. Chironomus varus limnaei Guibé, espèce jointive de Chironomus varus varus Gtgh. Bull. biol. Fr. Belg., 76 : 283-297.

Illies J. 1978. - Limnofauna Europaea. Eine Zusammenstellung aller die europäischen Binnengewässer bewohnenden mehrzellin'gen Tierarten mit Angaben über ihre Verbreitung und Ökologie. G. Fisher Verlag, Stuttgart : 532 p. + XVII + 1 carte.

Langton P.H. 1984. - A key to pupal exuviae of British Chironomidae. Privately published : Huntington, England : $324 \mathrm{p}$.

Langton P.H. 1991. - A key to pupal exuviae of West Palaearctic Chironomidae. Privately published : Huntington, England : $386 \mathrm{p}$.

Langton P.H. 1994. - A redescription of Parakiefferiella sp. D. Wülker, the pupa of Parakiefferiella wuelkeri Moubayed (Diptera : Chironomidae), a species new to Britain. Br.J. Ent. Nat. Hist., $7: 11-13$

Langton P.H. \& Cranston P.S. 1991. - Pupae in nomenclature and identification : West Palaearctic Orthocladius s. str. (Diptera : Chironomidae) revised. Systematic Entomology, $16: 239-252$.

Langton P.H., Cranston P.S. \& Armitage P. 1988. - The parthenogenetic midge of water supply systems, Paratanytarsus grimmii (Schneider) (Diptera : Chironomidae). Bul. ent. Res., $78: 317$ 328.

* (57a) Mercier L. \& Poisson R. 1923 (paru 1924). - Espèces nouvelles pour la faune de France observées dans le département du Calvados. Bull. Soc. Lin. Normandie, 7 série, 6: 160-168.

Moubayed J. 1991. - Male, female and pupa of Parakiefferiella pyrenaica sp. n. (Chironomidae, Orthocladiinae). Now. Rev. Ent. (N.S.), $8: 71-75$.

Moubayed J. 1994. - Parakiefferiella wuelkeri sp. n. (Diptera : Chironomidae) from western Europe and North Africa. Br. J. Ent. Nat. Hist., $7: 7-10$.

Oliver D.R. \& Dillon M.E. 1990. - A catalog of Nearctic Chironomidae. Research Branch Agriculture Canada, Public. 1857/B : 89p.

Pinder L.C. \& Reiss F. 1986. - The pupae of Chironominae (Diptera : Chironomidae) of the Holarctic region. Keys and diagnoses. Entomol. Scand., Suppl. n $28: 299-456$.

Reiss F. 1995. - Zur Identität von Parachironomus mauricii (Kruseman, 1933) (Chironomidae; Diptera). NachrBl. bayer. Ent., 44 : 57-60.

Reiss F. \& Reiff N. 1995. - Gesamtinventar der in Bayern nachgewiesenen Arten der Chironomidae (Insecta, Diptera, Nematocera). Lauterbomia. H. $21: 85-114$.

Reiss F. 1988. - Irmakia, ein neues Subgenus von Demicryptochironomus Lenz, 1941, mit der Beschreibung von vier neuen Arten. Spixiana, 11:1-12.

Saether O.A. 1977. - Taxonomic studies on Chironomidae : Nanocladius, Pseudochironomus and the Harnischia complex. Bull. Fish. Res. Bd Can., 196:143p.

Saether O.A. 1995. - Metriocnemus van der Wulp : seven species, revision of species, and new records (Diptera : Chironomidae). Annls. Limnol., $31: 35-64$.

Serra-Tosio B. \& Laville H. 1991. - Liste annotée des Diptères Chironomidés de France continentale et de Corse. Annls. Limnol., $27: 34-74$.

* (62a) Sinègre G., Babinot M., Vigo G. \& Tourenq J.N. 1990. Sensibilité de trois espèces de Chironomus (Diptera) à huit insecticides utilisés en démoustication. Annls. Limnol., $26: 65-71$.

* (62b) Strenzke K. 1960. - Metamorphose und Verwandschaftbeziehungen der Gattung Clunio Hal. (Dipt) (Terrestrische Chironomiden XXIV). Ann. Zool. Soc. Vanamo, 22(4): 1-30.

* Les références précédées d'un astérisque correspondent à la bibliographie oubliée dans Serra-Tosio \& Laville (1991). 